\title{
A Randomized Open label Phase-II Clinical Trial with or without Infusion of Plasma from Subjects after Convalescence of SARS- CoV-2 Infection in High-Risk Patients with Confirmed Severe SARS-CoV-2 Disease (RECOVER): A structured summary of a study protocol for a randomised controlled trial
}

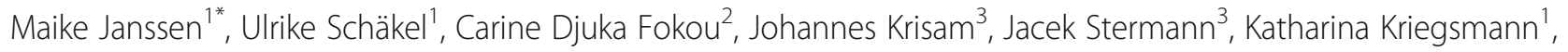
Isabella Haberbosch ${ }^{1}$, Jan Philipp Novotny ${ }^{1}$, Stefan Weber ${ }^{4}$, Maria Vehreschild ${ }^{4,5}$, Martin Bornhäuser ${ }^{6}$, Lars Bullinger ${ }^{7}$, Michael Schmitt ${ }^{1}$, Tobias Liebregts ${ }^{1}$, Peter Dreger ${ }^{1}$, Hanns-Martin Lorenz ${ }^{1}$, Anna Plaszczyca ${ }^{8}$, Ralf Bartenschlager ${ }^{8}$, Barbara Müller ${ }^{9}$, Hans-Georg Kräusslich ${ }^{9}$, Niels Halama ${ }^{10}$, Dirk Jäger ${ }^{10}$, Richard F. Schlenk ${ }^{1,2,10}$, Albrecht Leo ${ }^{11}$, Stefan Meuer ${ }^{11}$, Markus A. Weigand ${ }^{12}$, Johann Motsch ${ }^{12}$, Uta Merle ${ }^{13}$, Claudia M. Denkinger ${ }^{4}$ and Carsten Müller-Tidow ${ }^{1}$

\section{Abstract}

Objectives: Primary objectives

- To assess the time from randomisation until an improvement within 84 days defined as two points on a seven point ordinal scale or live discharge from the hospital in high-risk patients (group 1 to group 4) with SARS-CoV-2 infection requiring hospital admission by infusion of plasma from subjects after convalescence of SARS-CoV-2 infection or standard of care.

Secondary objectives

- To assess overall survival, and the overall survival rate at 2856 and 84 days.

(Continued on next page)

\footnotetext{
* Correspondence: maike.janssen@med.uni-heidelberg.de

'Department of Internal Medicine V, Heidelberg University Hospital, Heidelberg, Germany

Full list of author information is available at the end of the article
}

C C The Author(s). 2020 Open Access This article is licensed under a Creative Commons Attribution 4.0 International License, which permits use, sharing, adaptation, distribution and reproduction in any medium or format, as long as you give appropriate credit to the original author(s) and the source, provide a link to the Creative Commons licence, and indicate if changes were made. The images or other third party material in this article are included in the article's Creative Commons licence, unless indicated otherwise in a credit line to the material. If material is not included in the article's Creative Commons licence and your intended use is not permitted by statutory regulation or exceeds the permitted use, you will need to obtain permission directly from the copyright holder. To view a copy of this licence, visit http://creativecommons.org/licenses/by/4.0/ The Creative Commons Public Domain Dedication waiver (http://creativecommons.org/publicdomain/zero/1.0/) applies to the data made available in this article, unless otherwise stated in a credit line to the data. 
(Continued from previous page)

- To assess SARS-CoV-2 viral clearance and load as well as antibody titres.

- To assess the percentage of patients that required mechanical ventilation.

- To assess time from randomisation until discharge.

Trial design: Randomised, open-label, multicenter phase II trial, designed to assess the clinical outcome of SARSCoV-2 disease in high-risk patients (group 1 to group 4) following treatment with anti-SARS-CoV-2 convalescent plasma or standard of care.

Participants: High-risk patients $>18$ years of age hospitalized with SARS-CoV-2 infection in 10-15 university medical centres will be included. High-risk is defined as SARS-CoV-2 positive infection with Oxygen saturation at $\leq 94 \%$ at ambient air with additional risk features as categorised in 4 groups:

- Group 1, pre-existing or concurrent hematological malignancy and/or active cancer therapy (incl. chemotherapy, radiotherapy, surgery) within the last 24 months or less.

- Group 2, chronic immunosuppression not meeting the criteria of group 1.

- Group 3, age $\geq 50$ - 75 years meeting neither the criteria of group 1 nor group 2 and at least one of these criteria: Lymphopenia $<0.8 \times \mathrm{G} / \mathrm{l}$

and/or

D-dimer $>1 \mu \mathrm{g} / \mathrm{mL}$

- Group 4, age $\geq 75$ years meeting neither the criteria of group 1 nor group 2.

Observation time for all patients is expected to be at least 3 months after entry into the study. Patients receive convalescent plasma for two days (day 1 and day 2) or standard of care. For patients in the standard arm, cross over is allowed from day 10 in case of not improving or worsening clinical condition. Nose/throat swabs for determination of viral load are collected at day 0 and day 1 (before first CP administration) and subsequently at day $2,3,5,7,10,14,28$ or until discharge. Serum for SARS-Cov-2 diagnostic is collected at baseline and subsequently at day $3,7,14$ and once during the follow-up period (between day 35 and day 84).

There is a regular follow-up of 3 months. All discharged patients are followed by regular phone calls. All visits, time points and study assessments are summarized in the Trial Schedule (see full protocol Table 1).

All participating trial sites will be supplied with study specific visit worksheets that list all assessments and procedures to be completed at each visit. All findings including clinical and laboratory data are documented by the investigator or an authorized member of the study team in the patient's medical record and in the electronic case report forms (eCRFs).

Intervention and comparator: This trial will analyze the effects of convalescent plasma from recovered subjects with SARS-CoV-2 antibodies in high-risk patients with SARS-CoV-2 infection. Patients at high risk for a poor outcome due to underlying disease, age or condition as listed above are eligible for enrollment. In addition, eligible patients have a confirmed SARS-CoV-2 infection and $\mathrm{O}_{2}$ saturation $\leq 94 \%$ while breathing ambient air. Patients are randomised to receive (experimental arm) or not receive (standard arm) convalescent plasma in two bags (238 $337 \mathrm{ml}$ plasma each) from different donors (day 1, day 2). A cross over from the standard arm into the experimental arm is possible after day 10 in case of not improving or worsening clinical condition.

\section{Main outcomes: Primary endpoints:}

The main purpose of the study is to assess the time from randomisation until an improvement within 84 days defined as two points on a seven-point ordinal scale or live discharge from the hospital in high-risk patients (group 1 to group 4) with SARS-CoV-2 infection requiring hospital admission by infusion of plasma from subjects after convalescence of a SARS-CoV-2 infection or standard of care.

\section{Secondary endpoints:}

- Overall survival, defined as the time from randomisation until death from any cause 28-day, 56-day and 84-day overall survival rates.

- SARS-CoV-2 viral clearance and load as well as antibody titres.

- Requirement mechanical ventilation at any time during hospital stay (yes/no).

- Time until discharge from randomisation.

- Viral load, changes in antibody titers and cytokine profiles are analysed in an exploratory manner using paired non-parametric tests (before - after treatment).

(Continued on next page) 
(Continued from previous page)

Randomisation: Upon confirmation of eligibility (patients must meet all inclusion criteria and must not meet exclusion criteria described in section 5.3 and 5.4 of the full protocol), the clinical site must contact a centralized internet randomization system (https://randomizer.at/). Patients are randomized using block randomisation to one of the two arms, experimental arm or standard arm, in a 1:1 ratio considering a stratification according to the 4 risk groups (see Participants).

Blinding (masking): The study is open-label, no blinding will be performed.

Numbers to be randomised (sample size): A total number of 174 patients is required for the entire trial, $n=87$ per group.

Trial Status: Protocol version 1.2 dated 09/07/2020.

A recruitment period of approximately 9 months and an overall study duration of approximately 12 months is anticipated. Recruitment of patients starts in the third quarter of 2020.

The study duration of an individual patient is planned to be 3 months.

After finishing all study-relevant procedures, therapy, and follow-up period, the patient is followed in terms of routine care and treated if necessary.

\footnotetext{
Total trial duration:

Duration of the clinical phase:

First patient first visit (FPFV):

Last patient first visit (LPFV):

Last patient last visit (LPLV):

Trial Report completed:
}

18 months

12 months

$3^{\text {rd }}$ Quarter 2020

$2^{\text {nd }}$ Quarter 2021

$3^{\text {rd }}$ Quarter 2021

$4^{\text {th }}$ Quarter 2021

Trial registration: EudraCT Number: 2020-001632-10, https://www.clinicaltrialsregister.eu/ctr-search/trial/2020001632-10/DE, registered on 04/04/2020.

Full protocol: The full protocol is attached as an additional file, accessible from the Trials website (Additional file 1). In the interest in expediting dissemination of this material, the familiar formatting has been eliminated; this Letter serves as a summary of the key elements of the full protocol.

The study protocol has been reported in accordance with the Standard Protocol Items: Recommendations for Clinical Interventional Trials (SPIRIT) guidelines (Additional file 2).

The eCRF is attached (Additional file 3).

Keywords: COVID-19, Randomised controlled trial, protocol, convalescent plasma, early application, high-risk patients with severe disease

\section{Supplementary information}

Supplementary information accompanies this paper at https://doi.org/10. 1186/s13063-020-04735-y.

\section{Additional file 1. \\ Additional file 2. \\ Additional file 3.}

\section{Acknowledgements}

We express our sincere gratitude to the team of the Internal Medicine $\mathrm{V}$ Trials Office Jennifer Klemmer, Sabrina Zahn and Martina Gronkowski for their excellent technical support during trial implementation and conduct.

\section{Authors' contributions}

RFS: conception of the trial, lead writing of trial protocol, biostatistics, cocoordinating investigator. US, CDF, MV, MB, LB, MS, MAW, JM, PD, TL, HL, NH, $D J$ : writing of trial protocol and manuscript, co-coordinating investigators, conduct of the trial. CMT, CMD: conception of the trial, writing of trial protocol, principal investigator, conduct of the trial. JK, JS: conception of the trial, writing of trial protocol, biostatistician. MJ, IH, JPN, SW: sub- investigators conducting the trial and writing the manuscript. KK: biobanking, writing of trial protocol and lab manual. HGK, RB, AP, BM, NH: laboratory methods and evaluation. The authors read and approved the final manuscript.

\section{Funding}

The trial is co-financed by the BMBF program on emergency research funding for COVID-19 and the funds of Heidelberg University Hospital. The legal sponsor of the trial is the Ruprecht-Karls-University Heidelberg, Medical Faculty, represented in law by Heidelberg University Hospital, Germany, and its commercial director Katrin Erk. The trial is investigator-initiated, therefore, the main investigator has obtained all rights and duties from the legal sponsor except contract management. The legal sponsor itself does not have a role in collection, management, analysis, and interpretation of data; writing of the report; and the decision to submit the report for publication.

\section{Availability of data and materials}

After the trial has been completed and published, it is planned to make trial data available for re- and meta-analyses. An appropriate repository is defined at the end of the trial. 


\section{Ethics approval and consent to participate}

The study was approved by the responsible Ethics Committee of the Medical Faculty of Heidelberg University on 21/07/2020 (Afmo-280/2020) and from Paul-Ehrlich-Institute (PEI) on 14/07/2020 (407602). All patients are fully informed about the trial, are free to participate, and are asked to give their written consent prior to any trial-related procedures. We certify that this trial has received ethical approval from the appropriate Ethics Committee as described above

\section{Consent for publication}

Not applicable.

\section{Competing interests}

The authors declare that they have no competing interests.

\section{Author details}

'Department of Internal Medicine V, Heidelberg University Hospital, Heidelberg, Germany. ${ }^{2}$ NCT-Trial Center, National Center of Tumor Diseases, Heidelberg University Hospital and German Cancer Research Center, Heidelberg, Germany. ${ }^{3}$ Institute of Medical Biometry and Informatics, University of Heidelberg, Heidelberg, Germany. ${ }^{4}$ Division of Tropical Medicine, Department of Infectious Diseases, Heidelberg University Hospital, Heidelberg, Germany. ${ }^{5}$ Division of Infectious Diseases, University Hospital Frankfurt, Frankfurt, Germany. 'University Hospital Dresden, Dresden, Germany. ${ }^{7}$ Charité University Medicine, Berlin, Germany. ${ }^{8}$ Department of Infectious Diseases, Molecular Virology, Heidelberg University Hospital, Heidelberg, Germany. ${ }^{9}$ Department of Infectious Diseases, Virology, Heidelberg University Hospital, Heidelberg, Germany. ${ }^{10}$ Medical Oncology, National Center for Tumor Diseases (NCT), Heidelberg, Germany. ${ }^{11}$ Institute for Clinical Transfusion Medicine and Cell Therapy Heidelberg, Heidelberg, Germany. ${ }^{12}$ Department of Anaesthesiology, Heidelberg University Hospital, Heidelberg, Germany. ${ }^{13}$ Department of Internal Medicine IV, Heidelberg University Hospital, Heidelberg, Germany.

Received: 7 September 2020 Accepted: 8 September 2020 Published online: 06 October 2020

\section{Publisher's Note}

Springer Nature remains neutral with regard to jurisdictional claims in published maps and institutional affiliations.

Ready to submit your research? Choose BMC and benefit from:
- fast, convenient online submission
- thorough peer review by experienced researchers in your field
- rapid publication on acceptance
- support for research data, including large and complex data types
- gold Open Access which fosters wider collaboration and increased citations
- maximum visibility for your research: over 100M website views per year
At BMC, research is always in progress.
Learn more biomedcentral.com/submissions

